\title{
Giant Negative T Waves
}

Siva K. Kumar, MD, Rajesh M. Kabadi, MD, Paul J. Mather, MD

\section{Case Presentation}

A 62 year old Caucasian man with a past medical history significant for (long standing and controlled) HTN, lymphoma (diagnosed 2 yrs ago and currently in remission), and diffuse esophageal spasm presented to an outside hospital with expressive aphasia and right sided weakness. He was treated with tissue plasminogen activator (t-PA) for left MCA embolic stroke with excellent clinical response with very minimal residual expressive aphasia. Further work up and evaluation there revealed a large left ventricular apical mass consistent with thrombus and he was subsequently transferred to our facility for further evaluation and management of this apical mass.

\section{Hospital Course}

On presentation, the initial physical exam was significant only for an expressive aphasia. The presenting EKG, however (see Figure 1), showed diffusely negative $\mathrm{T}$ waves in all leads, especially in the left precordial leads.

The patient was asymptomatic and was initially started on anticoagulation with heparin to bridge him to a therapeutic INR on coumadin, given the report of thrombus. As part of a workup here to further delineate and look at this area of thrombus a transthoracic echocardiogram (see figure 2) was obtained. This was read as showing an apical mass which was reportedly $3.1 \times 1.6 \mathrm{~cm}$ in size.
Despite the echocardiogram findings, there was still doubt as to whether or not this was truly a case of apical thrombus, especially since these are fairly rare. This clinical thinking combined with the EKG findings, lead us to sequentially perform a nuclear stress test to evaluate for the possibility of a silent MI as a cause of this. This study was subsequently negative for any sign of ischemia. Finally, for definitive diagnosis, a cardiac MRI was performed to further evaluate the anatomy of the heart (see Figure 3). This showed marked focal left ventricular apical thickening of $9 \mathrm{~mm}$ likely corresponding to the abnormality seen on echocardiogram. This region of thickening demonstrates signal intensity similar to myocardium on all sequences and therefore is consistent with focal apical hypertrophy.

\section{Final Diagnosis}

Left Ventricular Atypical Apical Hypertrophic Cardiomyopathy (HCM)

\section{Discussion}

Apical HCM is an uncommon morphologic variant of HCM, and accounts for probably less than $10 \%$ of all cases. First discovered in Japanese males in 1976, it is most commonly seen in seen in Asia and has been reported in $41 \%$ of patients in China and $25 \%$ of patients in Japan. ${ }^{3}$

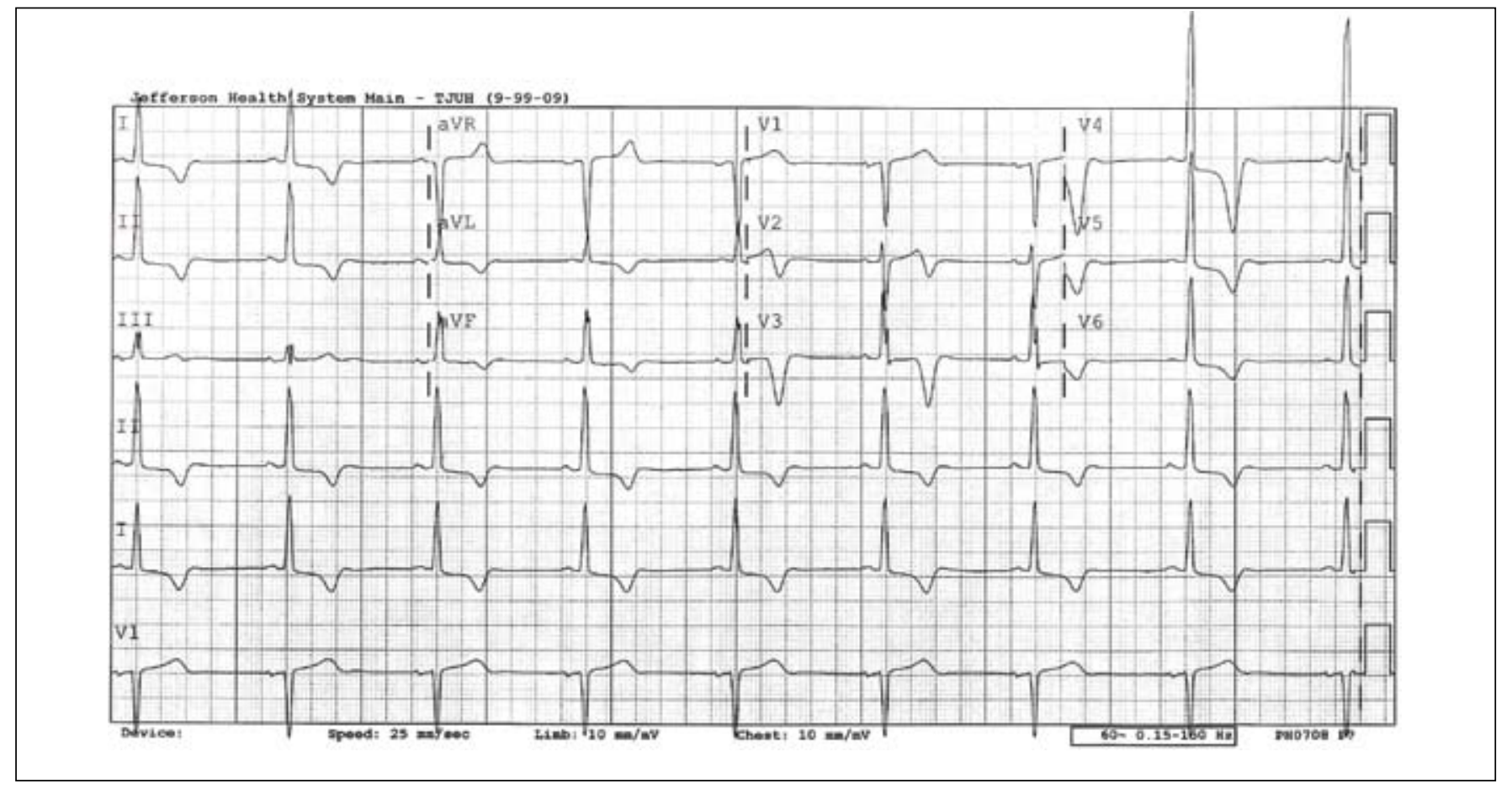

Figure 1. EKG-Normal sinus rhythm at the rate of 50 beats per minuts. Giant $T$ wave negativity was evident, particularly in the left precordial leads. 


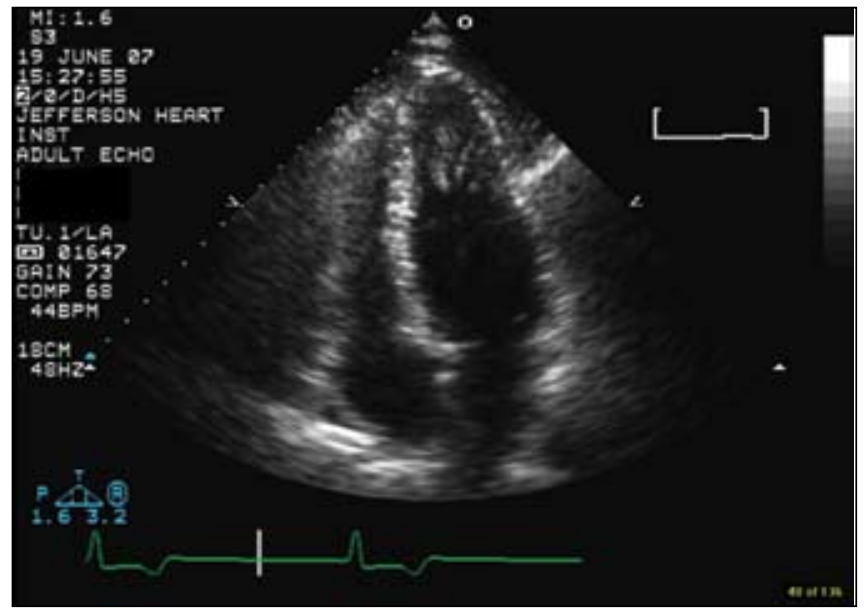

Figure 2. Transthoracic Echocardiogram. Overall normal left ventricular systolic function with segmental wall motion abnormalities. There is apical akinesis with a large $(3.1 \times 1.6 \mathrm{~cm})$ apical mass consistent with possible apical thrombus. A restrictive mitral inflow pattern is seen associated with significantly elevated left atrial pressure and reduced compliance of the left ventricle.

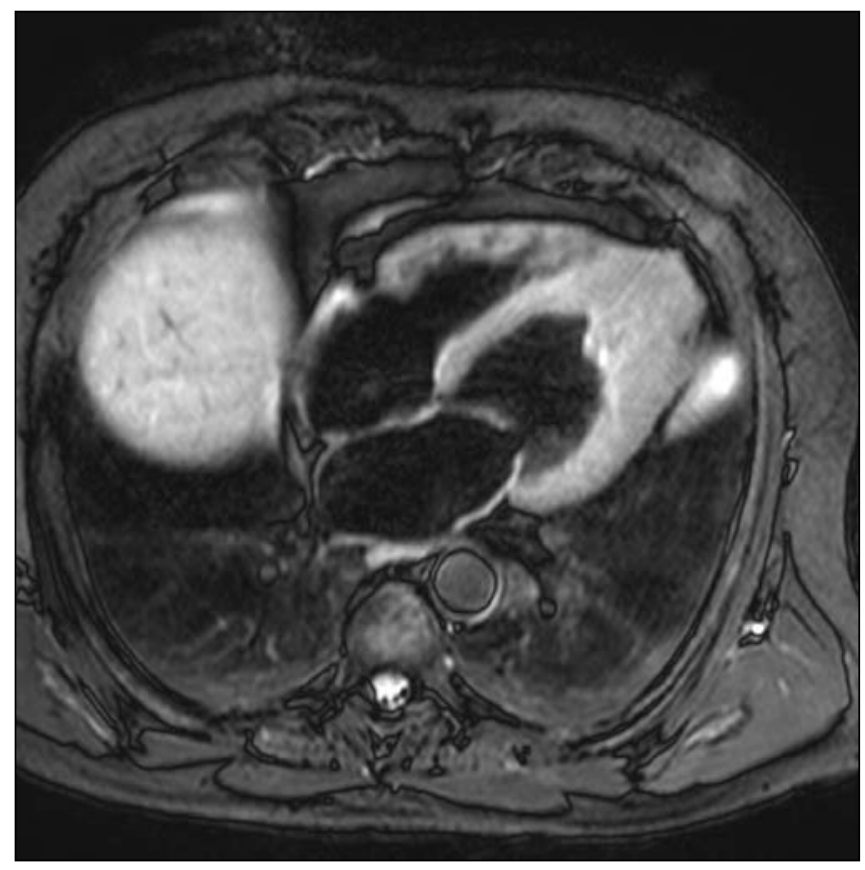

Figure 3. Cardiac MRI. Post-contrast T2 weighted image demonstrating myocardial uptake of contrast. A thrombus would not appear in such a manner as contrast material would not be taken up.
Typical presenting features consist of an audible and palpable fourth heart sound which reflects impaired ventricular relaxation. ECG features include "Giant" $T$ wave negativity in the left sided precordial leads, as was the case with our patient. Furthermore, imaging shows a "spade-like" configuration of the left ventricular cavity at end diastole. Some patients may also present with symptoms suspicious for ischemia such as chest pain and dyspnea on exertion. This combined with the above ECG findings will often lead to an immediate work up to rule out infarction.

There are multiple imaging techniques that can be used to detect apical HCM, with the first line being non-contrast echocardiography. The diagnostic accuracy of this modality is limited, however, by the fact that the apex can often be difficult to properly image. In many patients, the image seen may mimic akinesia or apical thrombus with poor acoustic windows, as was the case in our patient. In such cases, where the clinical suspicion is strong, measures such as cardiac MRI or echo with contrast may be more confirmatory. ${ }^{1}$ One study showed that in 100 affected patients, traditional echo made the correct diagnosis in $91 \%$ of patients, and the remaining nine were diagnosed using MRI. ${ }^{4}$ A study by Pons-Llabdo, et al showed that MRI allowed better overall assessment of the degree and extent of LVH than echo did. ${ }^{5}$ In this same study it was stated that MRI was able to properly visualize the apex in all patients, while echo was successful in only $61 \%$. Next to MRI, another useful modality is contrast echocardiography which may have better success than non-contrast with apical imaging. A case report presented by Acarturk et al demonstrated the value of contrast echo in diagnosing this condition. ${ }^{6}$ A final confirmatory sign which is often seen on diagnostic catheterization is that of the "spade like" configuration mentioned above. In most cases this is an incidental finding as the primary purpose of cardiac catheterization in this scenario is to evaluate for the possibility of concurrent coronary artery disease given the symptoms of chest pain and dyspnea on exertion that these patients will often present with. ${ }^{3}$ Patients with this form of HCM will often present at a young age and will be hospitalized due to symptoms and EKG criteria suspicious for coronary artery disease, so proper diagnosis can be of major importance. With a wall thickness of $9 \mathrm{~mm}$, our patient would be classified as having a mild form of this condition.

While the overall course of this condition is benign (especially in Asian countries), approximately $1 / 3$ of affected patients will experience serious cardiovascular events such as myocardial infarction or arrhythmia. In the asymptomatic patient with no significant ischemia or arrhythmia, no specific therapy has been outlined but counseling is certainly still recommended as a precaution. 4 Compared to patients with normal variant HCM, there is a much lesser incidence of sudden cardiac death. The 
mainstay of therapy is symptomatic monitoring, with patients being asked to inform their physician immediately in the event of syncope or presyncopal symptoms. Interestingly enough, there has been at least one published case of this condition being associated with a multi-organ syndrome which also includes atrial septal defect, hypothyroidism and renal failure. 2 It is unknown, at this time, whether or not this demonstrates any sort of defined syndrome.

\section{References}

1. Moon, JCC, Fisher NG, McKenna WJ, Pennell DJ, “Detection of Apical Hypertrophic Cardiomyopathy by cardiovascular magnetic resonance in patients with non-diagnostic echocardiography." Heart 2004;90:645-649.
2. Pattoneri, P., Giovannia P., Astorri, E., Borghetti, A., "Atrial hypertrophic cardiomyopathy and atrial septal defect: Part of a multi-organ syndrome?” Eur J Echocardiogr. 2007 Jun;8(3):226-9. Epub 2006 Mar 15.

3. Ting P, Gunasegaran K, Teo WS, "Electrocardiographical Case. Asymptomatic Patient with Deep T Wave Inversions.” Singapore Med Journal: 2007; 48(6), 586-89.

4. Eriksson MJ, Sonnenberg B, Woo A, Rakowski P, Parker TG, et al, "Long Term Outcome in Patients with Apical Hypertrophic Cardiomyopathy." Journal of the American College of Cardiology, Volume 39(4), 2002: 638-45.

5. Pons-Llado G, Carreras F, Borras X, Palmer J, Llauger J, et al. "Comparison of morphologic assessment of hypertrophic cardiomyopathy by magnetic resonance versus echocardiographic imaging." American Journal of Cardiology 1997; 79; 1651-6.

6. Acartürk E, Bozkurt A, Dönmez Y. Apical hypertrophic cardiomyopathy: diagnosis with contrast-enhanced echocardiography--a case report. Angiology. 